Journal of Mathematics and statistics 3 (3): 129-133, 2007

ISSN 1549-3644

(C) 2007 Science Publications

\title{
Sufficient Conditions for the Oscillation of Solutions of Some Nonlinear Parabolic Systems
}

\author{
S. Mazouzi and N. Ferfar \\ Department of Mathematics, University of Annaba, P.O. Box 12, Annaba 23000, Algeria
}

\begin{abstract}
We established some sufficient conditions for the oscillation of the solutions of some nonhomogenous nonlinear parabolic systems subject to Robin's boundary condition.
\end{abstract}

Key words: Oscillation, parabolic systems, positive solutions, Jensen's inequality, functional arguments

\section{INTRODUCTION}

We assume throughout this study that $\Omega$ is a bounded and smooth domain of $\mathrm{R}^{\mathrm{n}}$ with boundary $\partial \Omega$. First, we derive some useful sufficient conditions for oscillation of solutions of the following nonlinear parabolic problem

$$
\begin{aligned}
& \mathrm{u}_{\mathrm{t}}=\sum_{\mathrm{i}=1}^{\mathrm{n}} \mathrm{a}_{\mathrm{i}}(\mathrm{t}) \Delta \mathrm{u}\left(\mathrm{x}, \sigma_{\mathrm{i}}(\mathrm{t})\right)-\varepsilon \mathrm{b}(\mathrm{t}) \mathrm{f}\left(\mathrm{u}\left(\mathrm{x}, \sigma_{0}(\mathrm{t})\right)\right) \\
& -\varepsilon \mathrm{c}(\mathrm{t}) \int_{0}^{\mathrm{t}} \mathrm{k}(\mathrm{u}(\mathrm{x}, \mathrm{s})) \mathrm{ds}+\mathrm{d}(\mathrm{x}, \mathrm{t}), \forall(\mathrm{x}, \mathrm{t}) \in \Omega \times \mathrm{R}^{+} \\
& \frac{\partial \mathrm{u}}{\partial \eta}+\rho(\mathrm{x}, \mathrm{t}) \mathrm{u}=\psi(\mathrm{x}, \mathrm{t}), \text { on } \partial \Omega \times \mathrm{R}^{+} .
\end{aligned}
$$

We assume that $\rho(x, t)$ and $\psi(x, t)$ are realvalued continuous function on $\partial \Omega \times R^{+}$and $\rho(x, t)$ keeps a constant sign $\varepsilon=-1$ or $\varepsilon=+1$ on $\partial \Omega \times R^{+}$. Actually, our results of oscillation are not essentially based on the convexity of the nonlinearities $f(u)$ and $k(u)$ as it is the case in the works of ${ }^{[1-5]}$.

Next, we extend the above ideas to systems of nonlinear parabolic equations of the form

$$
\begin{aligned}
\mathrm{u}_{\mathrm{t}}= & \sum_{\mathrm{i}=1}^{\mathrm{n}} \mathrm{a}_{1 \mathrm{i}}(\mathrm{t}) \Delta \mathrm{u}\left(\mathrm{x}, \sigma_{\mathrm{i}}(\mathrm{t})\right)-\varepsilon_{1} \mathrm{~b}_{1}(\mathrm{t}) \mathrm{f}_{1}\left(\mathrm{v}\left(\mathrm{x}, \sigma_{0}(\mathrm{t})\right)\right) \\
& -\varepsilon_{1} \mathrm{c}_{1}(\mathrm{t}) \int_{0}^{\mathrm{t}} \mathrm{k}_{1}(\mathrm{v}(\mathrm{x}, \mathrm{s})) \mathrm{ds} \\
& +\mathrm{d}_{1}(\mathrm{x}, \mathrm{t}), \quad \forall(\mathrm{x}, \mathrm{t}) \in \Omega \times \mathrm{R}^{+} \\
\mathrm{v}_{\mathrm{t}}= & \sum_{\mathrm{i}=1}^{\mathrm{n}} \mathrm{a}_{2 \mathrm{i}}(\mathrm{t}) \Delta \mathrm{v}\left(\mathrm{x}, \tilde{\sigma}_{\mathrm{i}}(\mathrm{t})\right)-\varepsilon_{2} \mathrm{~b}_{2}(\mathrm{t}) \mathrm{f}_{2}\left(\mathrm{u}\left(\mathrm{x}, \tilde{\sigma}_{0}(\mathrm{t})\right)\right) \\
& -\varepsilon_{2} \mathrm{c}_{2}(\mathrm{t}) \int_{0}^{\mathrm{t}} \mathrm{k}_{2}(\mathrm{u}(\mathrm{x}, \mathrm{s})) \mathrm{ds} \\
& +\mathrm{d}_{2}(\mathrm{x}, \mathrm{t}), \quad \forall(\mathrm{x}, \mathrm{t}) \in \Omega \times \mathrm{R}^{+},
\end{aligned}
$$

subject to the boundary conditions

$$
\begin{aligned}
& \frac{\partial \mathrm{u}}{\partial \eta}+\rho_{1}(\mathrm{x}, \mathrm{t}) \mathrm{u}=\psi_{1}(\mathrm{x}, \mathrm{t}), \quad \text { on } \partial \Omega \times \mathrm{R}^{+}, \\
& \frac{\partial \mathrm{v}}{\partial \eta}+\rho_{2}(\mathrm{x}, \mathrm{t}) \mathrm{u}=\psi_{2}(\mathrm{x}, \mathrm{t}), \quad \text { on } \partial \Omega \times \mathrm{R}^{+},
\end{aligned}
$$

to obtain similar criteria for which the solution $(u, v)$ is oscillatory in $\Omega \times \mathrm{R}^{+}$.

Actually, these conditions are merely four functional differential systems of inequalities of first order which must not possess positive solutions. Finally, we give sufficient conditions for the nonexistence of eventually positive solutions of the latter systems.

Nonlinear parabolic equations: Before proceeding further we set the following assumptions:

(H1) $a_{i}(t)$ and $b(t)$ are nonnegative continuous functions defined on $R^{+}$, for $i=1, \ldots, n$.

(H2) $\sigma_{i}(t)$ are real-valued continuous defined on $R^{+}$which satisfy $\lim _{t \rightarrow+\infty} \sigma_{i}(t)=+\infty$, for $i=0,1, \ldots, n$.

(H3) $f(y)$ and $k(y)$ are odd, continuous in $\mathrm{R}$ and positive in $(0, \infty)$.

(H4) $\psi(x, t)$ and $\rho(x, t)$ are real-valued continuous functions on $\partial \Omega \times R^{+}$and the latter has a constant sign $\varepsilon$ on $\partial \Omega \times R^{+}$.

(H5) $d(x, t)$ is real-valued continuous function defined on $\Omega \times R^{+}$. 
We first need to define what we exactly mean by an oscillatory solution.

Definition 1: A solution $u$ of (1) is said to be oscillatory in $\Omega \times R^{+}$if, for every $t_{1}>0$, there exists some $\left(x_{0}, t_{0}\right) \in \Omega \times\left(t_{1}, \infty\right)$ such that $u\left(x_{0}, t_{0}\right)=0$, that is, $\mathrm{u}$ has arbitrarily large zeros in $\Omega \times R^{+}$.

Next, we define the functions $D(t)$ and $\Psi(t)$ as follows:

$D(t)=\int_{\Omega} d(x, t) d x$ and $\Psi(t)=\int_{\partial \Omega} \psi(x, t) d S$,

where $|\Omega|=\int_{\Omega} 1 d x$ and $d S$ is the Lebesgue measure on the boundary $\partial \Omega$.

Our first result is the following.

Theorem 1: Under the assumptions (H1)-(H5) a solution to the problem (1)-(2) is oscillatory in $\Omega \times R^{+}$if both of the functional differential inequalities (for $\delta= \pm 1$ )

$$
\begin{aligned}
\varepsilon\left(\frac{d}{d t} \int_{\Omega} u(x, t) d x\right. & \left.+\delta \sum_{i=1}^{n} a_{i}(t) \Psi\left(\sigma_{i}(t)\right)+\delta D(t)\right) \\
& +b(t) \int_{\Omega} f\left(u\left(x,\left(\sigma_{0}(t)\right)\right) d x\right. \\
& +c(t) \int_{0}^{t} \int_{\Omega} k(u(x, s)) d x d s \leq 0,
\end{aligned}
$$

have no eventually positive solution.

Proof: Suppose the contrary that problem (1)-(2) has a nonoscillatory solution $u$ in $\Omega \times R^{+}$. Suppose first that $u>0$ in $\Omega \times] t_{0}, \infty\left[\right.$, for some $t_{0}>0$. Integrating (1) over $\Omega$ and using the divergence theorem we get

$$
\begin{aligned}
\frac{d}{d t} \int_{\Omega} u(x, t) d x= & -\sum_{i=1}^{n} a_{i}(t) \int_{\partial \Omega} \rho u\left(x, \sigma_{i}(t)\right) d S \\
& +\sum_{i=1}^{n} a_{i}(t) \int_{\partial \Omega} \psi\left(x, \sigma_{i}(t)\right) d S \\
& -\varepsilon b(t) \int_{\Omega} f\left(u\left(x, \sigma_{0}(t)\right)\right) d x \\
- & \varepsilon c(t) \int_{0}^{t} \int_{\Omega} k(u(x, s)) d x d s+\int_{\Omega} d(x, t) d x,
\end{aligned}
$$

from which we infer

$$
\begin{aligned}
& \varepsilon\left(\frac{d}{d t} \int_{\Omega} u(x, t) d x-\sum_{i=1}^{n} a_{i}(t) \Psi\left(\sigma_{i}(t)\right)-D(t)\right) \\
& \quad+b(t) \int_{\Omega} f\left(u\left(x, \sigma_{0}(t)\right)\right) d x+c(t) \int_{0}^{t} \int_{\Omega} k(u(x, s)) d x d s \\
& =-\frac{1}{|\Omega|} \sum_{i=1}^{n} a_{i}(t) \int(|\rho| u)\left(x, \sigma_{i}(t)\right) d S .
\end{aligned}
$$

This clearly shows that (5) (for $\delta=-1$ ) has an eventually positive solution $u$ in $\Omega \times] t_{0}, \infty[$, which is a contradiction. Next, suppose that $u<0$ in $\Omega \times] t_{0}, \infty[$. Setting $u(x, t)=-v(x, t)$, we get the following problem

$$
\begin{gathered}
v_{t}=\sum_{i=1}^{n} a_{i}(t) \Delta v\left(x, \sigma_{i}(t)\right)-\varepsilon b(t) f\left(v\left(x, \sigma_{0}(t)\right)\right) \\
-\varepsilon c(t) \int_{0}^{t} k(v(x, s)) d s-d(x, t), \quad \forall(x, t) \in \Omega \times R^{+} \\
\frac{\partial v}{\partial \eta}+\rho(x, t) v=-\psi(x, t), \quad \text { on } \partial \Omega \times R^{+} .
\end{gathered}
$$

Reasoning as before we see that $\mathrm{v}$ is an eventually positive solution to (5) in $\Omega \times] t_{0}, \infty[$, (for $\delta=+1$ ). This is once again a contradiction which completes the proof of the Theorem.

Next, we have the following result of oscillation which is based on the convexity character of the function $f(u)$ and the kernel $k(u)$.

Theorem 2: Let $\mathrm{f}$ and $\mathrm{k}$ be convex in $] 0, \infty[$ and $\varepsilon=-1$. If both of the functional differential inequalities (for $\delta= \pm 1$ )

$$
\begin{gathered}
0 \leq \frac{d v(t)}{d t}-b(t) f\left(v\left(\sigma_{0}(t)\right)\right)-c(t) \int_{0}^{t} k(v(s)) d s \\
+\frac{\delta}{|\Omega|} \sum_{i=1}^{n} a_{i}(t) \Psi\left(\sigma_{i}(t)+\frac{\delta}{|\Omega|} D(t),\right.
\end{gathered}
$$

have no eventually positive solution, then the solution of problem (1)-(2) is oscillatory in $\Omega \times \mathrm{R}^{+}$.

Proof: Assume that $u>0$ in $\Omega \times] t_{0}, \infty\left[\right.$, for some $t_{0}>0$. Putting $\quad \tilde{u}(t)=\frac{1}{|\Omega|} \int_{\Omega} u(x, t) d x \quad$ and applying Jensen's Inequality we obtain

$$
\frac{1}{|\Omega|} \int_{\Omega} f\left(u\left(x, \sigma_{0}(t)\right)\right) d x \geq f\left(\widetilde{u}\left(\sigma_{0}(t)\right)\right) \text {. }
$$

Integrating (1) over $\Omega$ and dividing by $|\Omega|$, we get

$$
\begin{aligned}
0 \leq & -\frac{1}{|\Omega|} \sum_{i=1}^{n} a_{i}(t) \int_{\partial \Omega}(\rho u)\left(x, \sigma_{i}(t)\right) d s \\
\leq & \frac{d \widetilde{u}(t)}{d t}-b(t) f\left(\widetilde{u}\left(\sigma_{0}(t)\right)\right)-c(t) \int_{0}^{t} k(\widetilde{u}(s)) d s \\
& \quad-\frac{1}{|\Omega|} \sum_{i=1}^{n} a_{i}(t) \Psi\left(\sigma_{i}(t)\right)-\frac{1}{|\Omega|} D(t) .
\end{aligned}
$$

The latter functional differential inequality has $\widetilde{u}(t)$ as an eventually positive solution which contradicts the given assumption. 
Next, suppose that $\mathrm{u}=-\mathrm{v}<0$ in $\Omega \times] \mathrm{t}_{0}, \infty[$, for some $t_{0}>0$ and $\tilde{v}(t)=\frac{1}{|s|} \int_{\Omega} v(x, t) d x$, then

$$
\begin{aligned}
0 \leq & \frac{\mathrm{d} \tilde{\mathrm{v}}(\mathrm{t})}{\mathrm{dt}}-\mathrm{b}(\mathrm{t}) \mathrm{f}\left(\tilde{\mathrm{v}}\left(\sigma_{0}(\mathrm{t})\right)\right)-\mathrm{c}(\mathrm{t}) \int_{0}^{\mathrm{t}} \mathrm{k}(\tilde{\mathrm{v}}(\mathrm{s})) \mathrm{ds} \\
& +\frac{1}{|\Omega|} \sum_{\mathrm{i}=1}^{\mathrm{n}} \mathrm{a}_{\mathrm{i}}(\mathrm{t}) \Psi\left(\sigma_{\mathrm{i}}(\mathrm{t})\right)+\frac{1}{|\Omega|} \mathrm{D}(\mathrm{t}) .
\end{aligned}
$$

Thus, $\widetilde{v}(t)$ is an eventually positive solution to the functional differential equation (6) (corresponding to $\delta=+1)$ which leads to a contradiction. Therefore, the solution of problem (1)-(2) is oscillatory in $\Omega \times R^{+}$.

Now we are going to extend the foregoing results to systems of nonlinear parabolic equations.

Systems of nonlinear parabolic equations: In order to establish similar oscillation criteria for systems of nonlinear parabolic equations we set the following assumptions:

(H'1) The functions $\mathrm{b}_{1}(\mathrm{t}), \mathrm{b}_{2}(\mathrm{t}), \mathrm{c}_{1}(\mathrm{t}), \mathrm{c}_{2}(\mathrm{t}), \mathrm{a}_{\mathrm{li}}(\mathrm{t})$ and $\mathrm{a}_{2 \mathrm{i}}(\mathrm{t})$ are nonnegative and continuous in $\mathrm{R}^{+}$, for $i=1, \ldots, n$.

(H'2) The functions $d_{1}(x, t)$ and $d_{2}(x, t)$ are realvalued continuous in $\Omega \times \mathrm{R}^{+}$.

(H'3) The functions $\mathrm{f}_{\mathrm{i}}(\mathrm{y})$ and $\mathrm{k}_{\mathrm{i}}(\mathrm{y})$, for $\mathrm{i}=1,2$, are odd and continuous in $\mathrm{R}$ with $\mathrm{f}_{\mathrm{i}}(\mathrm{y})>0$ and $\mathrm{k}_{\mathrm{i}}(\mathrm{y})>0$, in $(0, \infty)$.

(H'4) The functions $\sigma_{i}(t)$ and $\tilde{\sigma}_{i}(t)$, for $i=0,1, \ldots, n$ are real-valued continuous functions in $R^{+}$with $\lim _{\mathrm{t} \rightarrow \infty} \sigma_{\mathrm{i}}(\mathrm{t})=\lim _{\mathrm{t} \rightarrow \infty} \tilde{\sigma}_{\mathrm{i}}(\mathrm{t})=+\infty$.

(H'5) $\rho_{\mathrm{i}}(\mathrm{x}, \mathrm{t})$ and $\psi_{\mathrm{i}}(\mathrm{x}, \mathrm{t})$, for $\mathrm{i}=1,2$, are realvalued continuous functions defined on $\partial \Omega \times \mathrm{R}^{+}$ and $\rho_{\mathrm{i}}(\mathrm{x}, \mathrm{t})$ has a constant sign $\varepsilon_{\mathrm{i}} \in\{-1,+1\}$ with $\varepsilon_{1}+\varepsilon_{2} \geq 0$.

Next, we shall use the following notations

$D_{i}(t)=\int_{\Omega} d_{1}(x, t) d x, \Psi_{i}(t)=\int_{\curvearrowright \Omega} \psi_{i}(x, t) d s, i=1,2$.

$\gamma_{0}=\min \left\{0, \operatorname{mininf}_{0 \leq i \leq n} \sigma_{i \geq 0}(t)\right\}$

and $\tilde{\gamma}_{0}=\min \left\{0, \operatorname{mininf}_{0 \leq i \leq n} \tilde{\sigma}_{i}(t)\right\}$
Before we state and prove our first result of oscillation of solutions of problem (3)-(4) we would like to give this definition.

Definition 2: A couple of functions $(u(x, t), v(x, t))$ such that $\mathrm{u}$ and $v \in C^{2}\left(\Omega \times\left(\gamma_{0}, \infty\right)\right) \cap C^{1}\left(\bar{\Omega} \times\left(\widetilde{\gamma}_{0}, \infty\right)\right)$ is said to be a solution to problem (3)-(4) if $\mathrm{u}$ and $\mathrm{v}$ satisfy equations (3) and the boundary conditions (4).

A solution $(u(x, t), v(x, t))$ to problem (3)-(4) is said to be oscillatory in $\Omega \times R^{+}$if either of $u(x, t)$ or $v(x, t)$ oscillates in $\Omega \times R^{+}$, in the sense that for every $t_{0} \geq 0$ there exists a point $\left(x_{1}, t_{1}\right) \in \Omega \times\left(t_{0}, \infty\right)$ such that $u\left(x_{1}, t_{1}\right)=0$ or $v\left(x_{1}, t_{1}\right)=0$.

Theorem 3: Let the assumptions (H'1)-(H'5) hold. If the 4 systems of functional differential inequalities corresponding to $(\alpha, \beta) \in\{-1,+1\} \times\{-1,+1\}$ :

$$
\begin{aligned}
& \varepsilon_{1}\left(\frac{d}{d t} \int_{\Omega} \mathrm{U}(\mathrm{x}, \mathrm{t}) \mathrm{dx}+\alpha \sum_{\mathrm{i}=1}^{\mathrm{n}} \mathrm{a}_{1 \mathrm{ii}}(\mathrm{t}) \Psi_{1}\left(\sigma_{\mathrm{i}}(\mathrm{t})\right)\right. \\
& \left.+\alpha \mathrm{D}_{1}(\mathrm{t})\right)+\alpha \beta \mathrm{b}_{1}(\mathrm{t}) \int_{\Omega} \mathrm{f}_{1}\left(\mathrm{~V}\left(\mathrm{x},\left(\sigma_{0}(\mathrm{t})\right)\right) \mathrm{dx}\right. \\
& +\alpha \beta \mathrm{c}_{1}(\mathrm{t}) \int_{0}^{\mathrm{t}} \int_{\Omega} \mathrm{k}_{1}(\mathrm{~V}(\mathrm{x}, \mathrm{s})) \mathrm{dxds} \quad \leq 0 \\
& \varepsilon_{2}\left(\frac{\mathrm{d}}{\mathrm{dt}} \int_{\Omega} \mathrm{V}(\mathrm{x}, \mathrm{t}) \mathrm{dx}+\beta \sum_{\mathrm{i}=1}^{\mathrm{n}} \mathrm{a}_{2 \mathrm{i}}(\mathrm{t}) \Psi_{2}\left(\tilde{\sigma}_{\mathrm{i}}(\mathrm{t})\right)\right. \\
& \left.+\beta \mathrm{D}_{2}(\mathrm{t})\right)+\alpha \beta \mathrm{b}_{2}(\mathrm{t}) \int_{\Omega} \mathrm{f}_{2}\left(\mathrm{U}\left(\mathrm{x},\left(\tilde{\sigma}_{0}(\mathrm{t})\right)\right) \mathrm{dx}\right. \\
& +\alpha \beta \mathrm{c}_{2}(\mathrm{t}) \int_{0}^{\mathrm{t}} \int_{\Omega} \mathrm{k}_{2}(\mathrm{U}(\mathrm{x}, \mathrm{s})) \mathrm{dxds} \quad \leq 0,
\end{aligned}
$$

have no eventually positive solution $(U(x, t), V(x, t))$ then the solution $(u(x, t), v(x, t))$ of the nonlinear parabolic system (3)-(4) is oscillatory in $\Omega \times R^{+}$.

Proof: To be clear, we say that a couple of functions $(U(x, t), V(x, t))$ is an eventually positive solution to (7) if there is $t_{0}>0$ such that $U(x, t)>0$ and $V(x, t)>0$, for every $x \in \Omega$ and $t>t_{0}$.

Suppose the contrary that systems (3)-(4) possesses a nonoscillatory solution $(u, v)$.

Suppose first that $u>0$ and $v>0$ in $\Omega \times] t_{0}, \infty[$, for some $t_{0}>0$. Using the divergence theorem in the first equation of (3) we get

$$
\begin{aligned}
& \frac{\mathrm{d}}{\mathrm{dt}} \int_{\Omega} \mathrm{u}(\mathrm{x}, \mathrm{t}) \mathrm{dx}=\sum_{\mathrm{i}=1}^{\mathrm{n}} \mathrm{a}_{1 \mathrm{i}}(\mathrm{t}) \Psi_{1}\left(\sigma_{\mathrm{i}}(\mathrm{t})\right) \\
& -\sum_{\mathrm{i}=1}^{\mathrm{n}} \mathrm{a}_{1 \mathrm{i}} \int_{\partial \Omega}\left(\mathrm{u} \rho_{1}\right)\left(\mathrm{x}, \sigma_{\mathrm{i}}(\mathrm{t})\right) \mathrm{ds} \\
& -\varepsilon_{1} \mathrm{~b}_{1}(\mathrm{t}) \int_{\Omega} \mathrm{f}_{1}\left(\mathrm{v}\left(\mathrm{x},\left(\sigma_{0}(\mathrm{t})\right)\right) \mathrm{dx}\right. \\
& -\varepsilon_{1} \mathrm{c}_{1}(\mathrm{t}) \int_{0}^{\mathrm{t}} \int_{\Omega} \mathrm{k}_{1}(\mathrm{v}(\mathrm{x}, \mathrm{s})) \mathrm{dxds}+\mathrm{D}_{1}(\mathrm{t}) .
\end{aligned}
$$




$$
\begin{aligned}
& \text { Hence } \\
& \begin{array}{l}
\varepsilon_{1}\left(\frac{d}{d t} \int_{\Omega} u(x, t) d x-\sum_{i=1}^{n} a_{1 i}(t) \Psi_{1}\left(\sigma_{i}(t)\right)-D_{1}(t)\right) \\
\quad+b_{1}(t) \int_{\Omega} f_{1}\left(v\left(x,\left(\sigma_{0}(t)\right)\right) d x\right. \\
\quad+c_{1}(t) \int_{0}^{t} \int_{\Omega} k_{1}(v(x, s)) d x d s \\
\quad=-\sum_{i=1}^{n} a_{1 i} \int_{\partial \Omega}\left(u\left|\rho_{1}\right|\right)\left(x, \sigma_{i}(t)\right) d S \leq 0 .
\end{array}
\end{aligned}
$$

Likewise we find that

$$
\begin{aligned}
& \varepsilon_{2}\left(\frac{d}{d t} \int_{\Omega} v(x, t) d x-\sum_{i=1}^{n} a_{2 i}(t) \Psi_{2}\left(\widetilde{\sigma}_{i}(t)\right)-D_{2}(t)\right) \\
& +b_{2}(t) \int_{\Omega} f_{2}\left(u\left(x,\left(\widetilde{\sigma}_{0}(t)\right)\right) d x\right. \\
& +c_{2}(t) \int_{0}^{t} \int_{\Omega} k_{2}(u(x, s)) d x d s \\
& \quad=-\sum_{i=1}^{n} a_{2 i} \int_{\partial \Omega}\left(v\left|\rho_{2}\right|\right)\left(x, \widetilde{\sigma}_{i}(t)\right) d S \leq 0 .
\end{aligned}
$$

Thus, the system (7), for $(\alpha, \beta)=(-1,-1)$, has a positive solution $(u, v)$ in $\Omega \times] t_{0}, \infty[$, which is contradiction.

Next, if we suppose that $u(x, t)=-\varphi(x, t)<0$ and $v(x, t)=-\xi(x, t)<0$ in $\Omega \times] t_{0}, \infty[$ for some $t_{0}>0$, then $(\varphi, \xi)$ satisfies the nonlinear parabolic system

$$
\begin{aligned}
& \varphi_{t}=\sum_{i=1}^{n} a_{1 i}(t) \Delta \varphi\left(x, \sigma_{i}(t)\right)-\varepsilon_{1} b_{1}(t) f_{1}\left(\xi\left(x, \sigma_{0}(t)\right)\right) \\
& \left.-\varepsilon_{1} c_{1}(t)\right]_{0}^{t} k_{1}(\xi(x, s)) d s-d_{1}(x, t), \forall(x, t) \in \Omega \times R^{+}, \\
& \xi_{t}=\sum_{i=1}^{n} a_{2 i}(t) \Delta \xi\left(x, \sigma_{i}(t)\right)-\varepsilon_{2} b_{2}(t) f_{2}\left(\varphi\left(x, \sigma_{0}(t)\right)\right) \\
& -\varepsilon_{2} c_{2}(t) \int_{0}^{t} k_{2}(\varphi(x, s)) d s-d_{2}(x, t), \forall(x, t) \in \Omega \times R^{+}, \\
& \left.\frac{\partial \varphi}{\partial \eta}+\rho_{1}(x, t) \varphi=-\psi_{1}(x, t), \quad \text { on } \partial \Omega \times\right] t_{0}, \infty[ \\
& \left.\frac{\partial \xi}{\partial \eta}+\rho_{2}(x, t) \xi=-\psi_{2}(x, t), \quad \text { on } \partial \Omega \times\right] t_{0}, \infty[
\end{aligned}
$$

Reasoning as before one gets

$$
\begin{gathered}
\varepsilon_{1}\left(\frac{d}{d t} \int_{\Omega} \varphi(x, t) d x+\sum_{i=1}^{n} a_{1 i}(t) \Psi_{1}\left(\sigma_{i}(t)\right)+D_{1}(t)\right) \\
+b_{1}(t) \int_{\Omega} f_{1}\left(\xi\left(x, \sigma_{0}(t)\right)\right) d x \\
\quad+c_{1}(t) \int_{0}^{t} \int_{\Omega} k_{1}(\xi(x, s)) d x d s \quad \leq 0
\end{gathered}
$$

and

$$
\begin{gathered}
\varepsilon_{2}\left(\frac{d}{d t} \int_{\Omega} \xi(x, t) d x+\sum_{i=1}^{n} a_{2 i}(t) \Psi_{2}\left(\widetilde{\sigma}_{i}(t)\right)+D_{2}(t)\right) \\
+b_{2}(t) \int_{\Omega} f_{2}\left(\varphi\left(x, \widetilde{\sigma}_{0}(t)\right)\right) d x \\
\quad+c_{2}(t) \int_{0}^{t} \int_{\Omega} k_{2}(\varphi(x, s)) d x d s \quad \leq 0 .
\end{gathered}
$$

Whence $(\varphi, \xi)$ is a positive solution to the functional differential system of inequalities (7) in $\Omega \times] t_{0}, \infty[$, for $(\alpha, \beta)=(1,1)$, this is once again a contradiction.

Finally, if $\quad u(x, t)=-\varphi(x, t)<0$ and $v(x, t)=\xi(x, t)>0 \quad$ (resp. $\quad u(x, t)=\varphi(x, t)>0$ and $v(x, t)=-\xi(x, t)<0$ in $\Omega \times] t_{0}, \infty\left[\right.$, for some $t_{0}>0$, then the positive solution $(\varphi, \xi)$ satisfies the functional differential system of inequalities (7) in $\Omega \times] t_{0}, \infty[$, for $(\alpha, \beta)=(1,-1)($ resp. $\quad(\alpha, \beta)=(-1,1)) . \quad$ This $\quad$ is a contradiction and the proof of the Theorem is complete.

Proposition 1: Suppose that assumptions (H'1)-(H'2) hold and either of

$$
\begin{aligned}
\limsup _{t \rightarrow+\infty} \int_{t_{0}}^{t} D_{1}(s) d s & =-\liminf _{t \rightarrow+\infty} \int_{t_{0}}^{t} D_{1}(s) d s \\
& =+\infty, \text { when } \varepsilon_{1}=+1,
\end{aligned}
$$

or

$$
\begin{aligned}
\limsup _{t \rightarrow+\infty} \int_{t_{0}}^{t} D_{2}(s) d s= & -\liminf _{t \rightarrow+\infty} \int_{t_{0}}^{t} D_{2}(s) d s \\
& =+\infty, \text { when } \varepsilon_{2}=+1,
\end{aligned}
$$

(for sufficiently large $t_{0}$ ). Then every solution of (3)-(4) is oscillatory in $\Omega \times] t_{0}, \infty[$.

Proof: Suppose that systems (7) have an eventually positive solution $(U(x, t), V(x, t))$ in $\Omega \times]_{1}, \infty\left[\right.$, for some $t_{1}>t_{0}$. Suppose that (8), then in particular for $\alpha=\beta=1$, (7) yields

$$
\begin{aligned}
& \frac{d}{d t} \int_{\Omega} U(x, t) d x+\sum_{i=1}^{n} a_{1 i}(t) \psi_{1}\left(\sigma_{i}(t)\right) \\
& \quad+b_{1}(t) \int_{\Omega} f_{1}\left(V\left(x,\left(\sigma_{0}(t)\right)\right) d x\right. \\
& \quad+c_{1}(t) \int_{0}^{t} \int_{\Omega} k_{1}(V(x, s)) d x d s \leq-D_{1}(t) .
\end{aligned}
$$

Hence

$\frac{d}{d t} \int_{\Omega} U(x, t) d x \leq-D_{1}(t), \quad t>t_{0}$,

from which we get by integration from $t_{0}$ to $t$

$\int_{\Omega} U(x, t) d x \leq \int_{\Omega} U\left(x, t_{0}\right) d x-\int_{t_{0}}^{t} D_{1}(s) d s, t>t_{0}$.

Therefore, 


$$
\begin{aligned}
0 \leq & \int_{\Omega} \lim _{t \rightarrow+\infty} \inf U(x, t) d x \leq \liminf _{t \rightarrow+\infty} \int_{\Omega} U(x, t) d x \\
& \leq-\limsup _{t \rightarrow+\infty} \int_{t_{0}}^{t} D_{1}(s) d s=-\infty
\end{aligned}
$$

This is a contradiction and so systems (7) have no eventually positive solutions in $\Omega \times R^{+}$. We conclude by Theorem 5 that problem (3)-(4) has an oscillatory solution $(u, v)$ in $\Omega \times R^{+}$.

\section{REFERENCES}

1. Liu, Z-R. and Y-H. Yu, 1995. Oscillation properties of solutions of neutral parabolic differential equations. Math. J. Toyama Univ., 18: 85-95.
2. Onose, H., 1996. On oscillation of nonlinear first order differential equations with its application. Proc. of 1st. World Congress 92, W. De gruyter, New York, pp: 1497-1504.

3. Onose, H., 1984. Oscillatory properties of the first order nonlinear advanced and delayed differential inequalities. Nonlinear Anal., 8: 171-180.

4. Yosida, N., 1986. Oscillation of nonlinear prarabolic equation with functional arguments. Hiroshima Math. J., 16: 305-314.

5. Yosida, N., 1995. On the oscillation of solutions to parabolic equations with functional arguments. Math. J. Toyama Univ., 18: 65-78. 